\title{
GAUSS-MARKOV PROCESSES ON HILBERT SPACES
}

\author{
BEN GOLDYS, SZYMON PESZAT, AND JERZY ZABCZYK
}

\begin{abstract}
K. Itô characterised in 1984 zero-mean stationary Gauss-Markov processes evolving on a class of infinite-dimensional spaces. In this work we extend the work of Itô in the case of Hilbert spaces: Gauss-Markov families that are time-homogenous are identified as solutions to linear stochastic differential equations with singular coefficients. Choosing an appropriate locally convex topology on the space of weakly sequentially continuous functions we also characterize the transition semigroup, the generator and its core, thus providing an infinite-dimensional extension of the classical result of Courrège in the case of Gauss-Markov semigroups.
\end{abstract}

\section{Contents}

1. Introduction

2. Main results

3. Proof of Theorem 2.4

4. Proof of Theorem 2.7

5. Proof of Theorem 2.8

6. Example: Heat equation with boundary noise

Appendix A. Proof of Lemma 2.3

Acknowledgement

References

\section{INTRODUCTION}

Let $\{\mu(t, x): x \in H, t \geq 0\}$ be a family of Gaussian transition kernels on a real separable Hilbert space $\left(H,\langle\cdot, \cdot\rangle_{H}\right)$, that is,

$$
\mu(t, x)=\mathscr{N}(m(t, x), Q(t, x)),
$$

and for every Borel set $\Gamma \subset H$, the functions $x \mapsto \mu(t, x)(\Gamma), t \geq 0$, are measurable, and

$$
\mu(s+t, x)(\Gamma)=\int_{H} \mu(s, x)(\mathrm{d} y) \mu(t, y)(\Gamma), \quad s, t \geq 0, x \in H .
$$

Received by the editors July 9, 2013 and, in revised form, October 19, 2013.

2010 Mathematics Subject Classification. Primary 60G15, 60H15, 60 J99.

Key words and phrases. Gauss-Markov process, Ornstein-Uhlenbeck process, Gaussian measure, $b w$-topology, strict topology, generator.

The work of the first author was partially supported by the ARC Discovery Grant DP120101886. Part of this work was prepared during his visit to the Institute of Mathematics of the Polish Academy of Sciences. He gratefully acknowledges the excellent working conditions and stimulating atmosphere of the Institute.

The work of the second and third authors was supported by Polish National Science Center grant DEC2013/09/B/ST1/03658. 
It is well known (see for example Theorem 8.4 in [17]) that there exist a measurable space $(\Omega, \mathscr{F})$ endowed with a filtration $\left(\mathscr{F}_{t}\right)$, an $H$-valued and $\left(\mathscr{F}_{t}\right)$-adapted stochastic process $Z$ defined on $H$, and a family of probability measures $\left\{\mathbb{P}^{x}: x \in H\right\}$ on $\Omega$ such that for every $x \in H$ the process $Z$ is Markov on the probability space $\left(\Omega, \mathscr{F},\left(\mathscr{F}_{t}\right), \mathbb{P}^{x}\right)$, and its transition kernel is $\mu(t, x)$ with

$$
m(0, x)=\mathbb{P}^{x}(Z(0)=x)=1, \quad x \in H .
$$

Let us recall that the system $\left(\Omega, \mathscr{F},\left(\mathscr{F}_{t}\right), Z,\left\{\mathbb{P}^{x}: x \in H\right\}\right)$ is called a Markov family with transition kernels $\mu(t, x), x \in H, t \geq 0$, and is said to be a Gauss-Markov family if the process $Z$ is Gaussian under each measure $\mathbb{P}^{x}$.

The aim of this paper is to characterise such processes. Our Theorem 2.4 describes the structure of the transition semigroup, Theorem 2.7 provides a construction of a stochastic equation which the process satisfies and Theorem 2.8 characterises the generator of the transition semigroup. In particular, we will prove that there exist

- a $C_{0}$-semigroup $(L(t))$ (with the generator $A$ ) acting on $H$,

- a Hilbert space $V$ in which $H$ is densely and continuously embedded and such that the semigroup $(L(t))$ can be extended to a $C_{0}$-semigroup $\left(L_{V}(t)\right)$ on $V$,

- a vector $b_{V} \in V$ and a bounded linear operator $B: H \rightarrow V$,

such that for all $h, g \in V^{\prime} \hookrightarrow H^{\prime} \equiv H$, and $t \geq 0$ and $x \in H$, the mean value $m(t, x)$ and covariance $Q(t, x)$ satisfy

$$
\langle m(t, x), h\rangle_{H}=\langle L(t) x, h\rangle_{H}+\int_{0}^{t}\left(L_{V}(s) b_{V}, h\right) \mathrm{d} s
$$

and

$$
\langle Q(t, x) h, g\rangle_{H}=\int_{0}^{t}\left\langle B^{\star} L_{V}^{\star}(s) h, B^{\star} L_{V}^{\star}(s) g\right\rangle_{H} \mathrm{~d} s .
$$

In the formulae above we used the notation $(\cdot, \cdot)$ for the canonical bilinear form on $V \times V^{\prime}$ which coincides with the scalar product $\langle\cdot, \cdot\rangle_{H}$ on $H \times V^{\prime}$.

Having the above form of $m$ and $Q$, we prove that given a Markov family $\left(\Omega, \mathscr{F},\left(\mathscr{F}_{t}\right), Z\right),\left\{\mathbb{P}^{x}: x \in H\right\}$ with transition kernels $\{\mu(t, x): x \in H, t \geq 0\}$, there is a generalized process $W$ on the Hilbert space $H_{0}:=\overline{\operatorname{Im~} B^{\star}}$ such that under each $\mathbb{P}^{x}, W$ is cylindrical on $H_{0}$ (see Definition 2.6) and $Z$ is the mild solution (see (2.6)) to the equation

$$
\mathrm{d} Z=\left(A Z+b_{V}\right) \mathrm{d} t+B \mathrm{~d} W .
$$

The above characterisation is an extension of the work of K. Itô, who obtained in [16] a similar representation for a stationary Gauss-Markov process with zero mean. Since we do not assume stationarity and we work with a Gauss-Markov family of processes instead of a single process, the approach of [16] cannot be adopted and a different argument is required to derive representations for $m(t, x)$ and $Q(t, x)$.

Our third, purely analytic result is in the spirit of a theorem of Ph. Courrège [4]. Namely, Courrège obtained a representation of a generator for an arbitrary Markov semigroup that is strongly continuous in $C_{0}\left(\mathbb{R}^{d}\right)$ 1] and such that $C_{c}^{\infty}\left(\mathbb{R}^{d}\right)$ is a core for its generator. It is well known (see for example [6]) that the space $C_{b}^{\text {norm }}(H)$

\footnotetext{
${ }^{1}$ We use standard notation $C_{0}\left(\mathbb{R}^{d}\right)$ for the space of continuous functions vanishing at infinity and $C_{c}^{\infty}\left(\mathbb{R}^{d}\right)$ for the space of infinitely differentiable functions with compact support.
} 
of bounded continuous functions on $H$ endowed with the supremum norm is not appropriate for the analysis of Markovian semigroups on Hilbert spaces. In order to obtain a counterpart of the Courrège theorem we need to introduce two locally convex topologies. The first one is the bounded weak topology on $H$ defined in 13 . It was demonstrated in [20] and recently in [3] that, in fact, it is a natural concept for Markovian transition semigroups and particularly useful for proving the existence of an invariant measure. We will use the notation $H_{b w}$ for the space $H$ endowed with the bounded weak topology, $C_{b}\left(H_{b w}\right)$ for the space of bounded continuous functions on $H_{b w}$, and $C_{b}^{n o r m}\left(H_{b w}\right)$ for the space $C_{b}\left(H_{b w}\right)$ endowed with the norm topology. It was shown in [20] that $C_{b}^{n o r m}\left(H_{b w}\right)$ is precisely the space of bounded functions that are weakly sequentially continuous. Another locally convex topology, known as the strict topology, will be introduced on the space $C_{b}\left(H_{b w}\right)$. The strict topology can be defined as the strongest topology on $C_{b}\left(H_{b w}\right)$ which on norm bounded sets of $C_{b}^{n o r m}\left(H_{b w}\right)$ coincides with the topology of uniform convergence on compacts of $H_{b w}$. For alternative definitions of this topology and its applications to the theory of Markov semigroups, see [15] and the references therein.

In this paper we show that the transition semigroup

$$
P_{t} \phi(x)=\int_{H} \phi(y) \mu(t, x)(\mathrm{d} y)
$$

is strongly continuous in $C_{b}^{\text {strict }}\left(H_{b w}\right)$. Moreover, we will identify a core of the generator $\mathscr{G}$ of $\left(P_{t}\right)$ in $C_{b}^{\text {strict }}\left(H_{b w}\right)$ and will derive an explicit formula for the generator acting on functions from the core; see Theorem 2.8. These results provide a version of the Courrège theorem for a Gauss-Markov semigroup in a Hilbert space.

Although the extension concerns a rather narrow class of transition semigroups, we believe that it does suggest how a general infinite-dimensional version of the Courrège theorem should look.

If the state space $H$ is one-dimensional, then results of our paper are part of the mathematical folklore. More generally, it is not very difficult to obtain our result if $\operatorname{dim}(H)<\infty$. Let us note here that in finite-dimensional spaces a similar problem is considered in the framework of affine processes; see for example [12. In the theory of affine models a much wider class of processes is considered, but the linearity of the function $x \mapsto m(t, x)$ and the fact that $x \mapsto Q(t, x)$ is constant are assumed from the very beginning while we do derive these properties as a result of a careful analysis.

In infinite dimensions the only result in this direction was obtained by Itô in [16. Let us also recall a related problem to characterise a family of measures that satisfy the so-called skew convolution equation [22. In that paper it is assumed again from the very beginning that the expectation $m(t, x)$ is linear in $x$ and the covariance $Q(t, x)$ is constant in $x$.

Main results are formulated in Section 2, The proofs are presented in the following sections. In the final section, Section 6, we discuss an application of our results to models with boundary noise.

\section{MAin RESUlts}

Let $\left(\Omega, \mathscr{F},\left(\mathscr{F}_{t}\right), Z,\left\{\mathbb{P}^{x}: x \in H\right\}\right)$ be a Gauss-Markov family with transition kernels $\mu(t, x), x \in H, t \geq 0$; that is, the process $Z$ is Gaussian under the measure $\mathbb{P}^{x}$ 
for every $x \in H$. By definition we have

$$
m(t, x)=\mathbb{E}^{x} Z(t), \quad x \in H, t \geq 0,
$$

where $\mathbb{E}^{x}$ is the operator of mathematical expectation with respect to $\mathbb{P}^{x}$. Let $Q(s, t, x)$ denote the covariance operator of the process $Z$ under the probability $\mathbb{P}^{x}$; that is, for $h, k \in H$,

$$
\langle Q(s, t, x) h, k\rangle_{H}=\mathbb{E}^{x}\langle Z(s)-m(s, x), h\rangle_{H}\langle Z(t)-m(t, x), k\rangle_{H} \cdot
$$

If $s=t$, then we put

$$
Q(t, x)=Q(t, t, x), \quad x \in H, t \geq 0 .
$$

Let $\phi: H \rightarrow \mathbb{R}$ be continuous and such that $|\phi(x)| \leq C\left(1+|x|_{H}^{2}\right)$. Then for all $s, t \geq 0$ and $x, y \in H$, the Markov property yields

$$
\mathbb{E}^{y}(\phi(Z(t+s)) \mid Z(s)=x)=\mathbb{E}^{x} \phi(Z(t)) .
$$

The following hypothesis is a standing assumption of the rest of the paper.

Hypothesis 2.1. (1) For every $x \in H$ and $h, k \in H$ the functions

$$
t \mapsto\langle m(t, x), h\rangle_{H} \quad \text { and } \quad t \mapsto\langle Q(t, x) h, k\rangle_{H}
$$

are continuous on $[0, \infty)$.

(2) For any $t \geq 0$ and $h, k \in H$ the functions

$$
x \rightarrow\langle m(t, x), h\rangle_{H} \quad \text { and } \quad x \rightarrow\langle Q(t, x) h, k\rangle_{H}
$$

are continuous on $H$.

(3) For any $t>0$ and any $x \in H$,

$$
\overline{\operatorname{Im} Q(t, x)}=H \text {. }
$$

We note that in view of (1.3), Hypothesis 2.1 yields for every $x \in H$ :

$$
\lim _{t \downarrow 0}\langle m(t, x), h\rangle_{H}=\langle x, h\rangle_{H}, \quad h \in H,
$$

and

$$
\lim _{t \downarrow 0}\langle Q(t, x) h, k\rangle_{H}=0, \quad h, k \in H .
$$

In order to formulate our results we need some preparations. Let $L=(L(t))$ be any $C_{0}$-semigroup on $H$ and let $A$ denote its generator. Then for $\lambda>0$ big enough $(\lambda-A)$ is boundedly invertible on $H$; hence a new norm on $H$ can be defined by the formula

$$
|x|_{-1}:=\left|(\lambda-A)^{-1} x\right|_{H}, \quad x \in H .
$$

Definition 2.2. The space $V$ associated with $L$ is the Hilbert space being the completion of $H$ with respect to the norm $|\cdot|_{-1}$.

Clearly, the topological space $V$ does not depend on $\lambda>0$. Under identification $H^{\prime} \equiv H$ we have $V^{\prime} \hookrightarrow H^{\prime} \equiv H \hookrightarrow V$. We denote by $(\cdot, \cdot)$ the canonical bilinear form on $V \times V^{\prime}$. We will need the following technical lemma. Its proof is postponed to Appendix $\mathrm{A}$ 
Lemma 2.3. ( $i)$ The semigroup $(L(t))$ extends to a $C_{0}$-semigroup $\left(L_{V}(t)\right)$ on $V$ with the generator $A_{V}$ whose domain dom $A_{V}=H$. Moreover, $A_{V}$ is a bounded linear operator from $H$ to $V$.

(ii) $V^{\prime}=\operatorname{dom} A^{\star}$, where $A^{\star}$ is the adjoint operator to $A$ in the space $H$ identified with its dual $H^{\prime}$. Moreover, $A^{\star}$ is a bounded operator from $V^{\prime}$ to $H$ and $A^{\star}=\left(A_{V}\right)^{\star}$. Finally, for any $t \geq 0, L_{V}^{\star}(t)$ is the restriction of $L^{\star}(t)$ to $V^{\prime}$.

(iii) Let $S: V^{\prime} \rightarrow V$ be a symmetric, non-negative operator. Then there is a bounded operator $B: H \rightarrow V$ such that $S=B B^{\star}$.

We can formulate now our first main result. It gives a precise description of the mappings $m$ and $Q$ and will also play the crucial role in the proofs of Theorems 2.7 and 2.8 below. The proof of Theorem 2.4 is postponed to Section 3 . Below $L_{V}^{\star}(s)$ and $B^{\star}$ are adjoint operators to $L_{V}(t)$ and $B$, where the dual space $V^{\prime}$ to $V$ is identified by the scalar product on $H$. Thus $L_{V}^{\star}(t)$ is a bounded linear operator on $V^{\prime}$, and $B^{\star}$ is a bounded linear operator from $V^{\prime}$ to $H^{\prime} \equiv H$.

Theorem 2.4. There exist a strongly continuous semigroup $(L(t))$ with the generator $A$ on $H$, the space $V$ associated with $L$, a vector $b_{V} \in V$, and a bounded linear operator $B: H \rightarrow V$ such that for all $t \geq s \geq 0, x \in H$, and $h, g \in V^{\prime}$, the mean-value $m(t, x)$ is determined by (1.4). The operator $Q(s, t):=Q(s, t, x)$ is independent of $x \in H$, and

$$
Q(s, t, x)=L(t-s) Q(s), \quad 0 \leq s \leq t, x \in H .
$$

In particular, the covariance operator $Q(t):=Q(t, x)$ is independent of $x \in H$ and is determined by (1.5).

Moreover, for any $t>0$, the operator $B^{\star} L_{V}^{\star}(t): V^{\prime} \rightarrow H$ has a (unique) extension to a Hilbert-Schmidt operator (still denoted by $\left.B^{\star} L_{V}^{\star}(t)\right)$ from $H$ to $H$, and

$$
\int_{0}^{T}\left\|B^{\star} L_{V}^{\star}(t)\right\|_{L_{(H S)}(H, H)}^{2} \mathrm{~d} t<\infty, \quad \forall T>0 .
$$

Finally

$$
Q(T)=\int_{0}^{T}\left(B^{\star} L_{V}^{\star}(t)\right)^{\star} B^{\star} L_{V}^{\star}(t) \mathrm{d} t<\infty, \quad \forall T>0,
$$

where the integral is considered in the Bochner sense in the space of nuclear (or trace-class) operators on $H$.

Remark 2.5. Let $t \geq 0$ and $h \in V^{\prime}$. By (1.4),

$$
\int_{0}^{t}\left(L_{V}(s) b_{V}, h\right) \mathrm{d} s=\left(\int_{0}^{t} L_{V}(s) b_{V} \mathrm{~d} s, h\right)=\langle m(t, x)-L(t) x, h\rangle_{H} .
$$

Since $V^{\prime}$ is dense in $H$, we have

$$
\int_{0}^{t} L_{V}(s) b_{V} \mathrm{~d} s=m(t, x)-L(t) x \in H, \quad \forall t \geq 0 .
$$

For any $t>0, L_{V}(t) B$ is a bounded linear operator from $H$ to a bigger space $V$. Since

$$
L_{V}(t) B=\left(B^{\star} L_{V}^{\star}(t)\right)^{\star},
$$


it is in fact a Hilbert-Schmidt operator on $H$. Moreover,

$$
\int_{0}^{T}\left\|L_{V}(t) B\right\|_{L_{(H S)}(H, H)}^{2} \mathrm{~d} t<\infty, \quad \forall T>0 .
$$

In the remaining part of this section $(L(t)), V, b_{V}$, and $B$ are as in Theorem 2.4. For the convenience of the reader we recall below the definition of a cylindrical Wiener process. For the theory of stochastic integration with respect to it we refer the reader to 8$]$.

Definition 2.6. Let $(\Omega, \mathscr{F})$ be a measurable space, and let $E$ be a Hilbert space. A family of linear mappings $W(t), t \geq 0$, from $E$ to the space of measurable real-valued functions on $(\Omega, \mathscr{F})$ is called a generalised random process on $E$. Assume now that $\mathbb{P}$ is a probability measure and $\left(\mathscr{F}_{t}\right)$ is a filtration on $(\Omega, \mathscr{F})$. Then a generalised random process $W$ is a cylindrical Wiener on $E$ defined on the probability space $\left(\Omega, \mathscr{F},\left(\mathscr{F}_{t}\right), \mathbb{P}\right)$ if:

(i) for any finite subsets $\left\{x_{i}: i=1, \ldots, n\right\} \subset E$ and $\left\{t_{i}: i=1, \ldots, n\right\} \subset$ $[0,+\infty)$, the random vector $\left(W\left(t_{1}\right)\left[x_{1}\right], \ldots, W\left(t_{n}\right)\left[x_{n}\right]\right)$ is Gaussian in $\mathbb{R}^{n}$;

(ii) for each $x \in E$ such that $|x|_{E}=1$, the process $(W(t)[x]: t \geq 0)$ is a standard Wiener process with respect to $\left(\mathscr{F}_{t}\right)$;

(iii) for all $x, y \in E$ and $t, s \geq 0, \mathbb{E} W(t)[x] W(s)[y]=t \wedge s\langle x, y\rangle_{E}$.

A cylindrical Wiener process $W$ on $E$ can be identified with

$$
W(t)=\sum_{k} W_{k}(t) e_{k}, \quad t \geq 0,
$$

where $\left(e_{k}\right)$ is an orthonormal basis of $E$ and $\left(W_{k}\right)$ is a sequence of independent real-valued Wiener processes on $(\Omega, \mathscr{F},(\mathscr{F} t), \mathbb{P})$; see 8 for more details.

Now we can formulate our second main result. Its proof is postponed to Section 4. In the formulation of the result $H_{0}=\overline{\operatorname{Im} B^{\star}}$ is the closed subspace of $H$ equipped with the scalar product inherited from $H$.

Theorem 2.7. Let $\left(\Omega, \mathscr{F},\left(\mathscr{F}_{t}\right), Z,\left\{\mathbb{P}^{x}: x \in H\right\}\right)$ be a Gauss-Markov family with the transition kernels $\{\mu(t, x): x \in H, t \geq 0\}$. Then there exists a generalised process $W$ on the Hilbert space $H_{0}$ such that for any $x \in H, W$ is a cylindrical Wiener process on $H_{0}$ defined on $\left(\Omega, \mathscr{F},\left(\mathscr{F}_{t}\right), \mathbb{P}^{x}\right)$ and such that for every $x \in H$ we have

$$
Z(t)=L(t) x+\int_{0}^{t} L_{V}(t-s) b_{V} \mathrm{~d} s+\int_{0}^{t} L_{V}(t-s) B \mathrm{~d} W(s), \quad t \geq 0, \mathbb{P}^{x}-a . s .
$$

Our final result is an extension of the Courrège theorem. We introduce first certain convenient locally convex topologies.

In the terminology of [13] the bounded weak topology $\tau^{b w}$ on $H$ is the strongest locally convex topology on $H$ that coincides with the weak topology on every ball. We will denote by $H_{b w}$ the space $H$ endowed with the topology $\tau^{b w}$. It has been proved in 20] that $\phi \in C_{b}\left(H_{b w}\right)$ if and only if $\phi$ is bounded and weakly sequentially continuous on $H$.

Let $\tau^{n}$ and $\tau^{c}$ denote, respectively, the norm topology and the topology of the uniform convergence on compacts on the space $C_{b}\left(H_{b w}\right)$. The strict topology $\tau^{s}$ is defined as the strongest locally convex topology on $C_{b}\left(H_{b w}\right)$ that coincides with the topology $\tau^{c}$ on compacts. We will say that $\phi \in \mathscr{F} C_{b}^{\infty}\left(A^{\star}\right)$ if there exist $n \geq 1$, $f \in C_{b}^{\infty}\left(\mathbb{R}^{n}\right)$ and $h_{1}, \ldots, h_{n} \in V^{\prime}=\operatorname{dom} A^{\star}$ such that

$$
\phi(x)=f\left(\left\langle x, h_{1}\right\rangle_{H}, \ldots,\left\langle x, h_{n}\right\rangle_{H}\right)
$$


and

$$
\sup _{x \in H}\left|\left\langle x, A^{\star} D \phi(x)\right\rangle_{H}\right|<\infty .
$$

It can be easily shown that $\mathscr{F} C_{b}^{\infty}\left(A^{\star}\right)$ is dense in $C_{b}^{\text {strict }}\left(H_{b w}\right)$. The proof of the result below is postponed to Section 5 . In the theorem $\left(P_{t}\right)$ is the semigroup defined by (1.7).

Theorem 2.8. The semigroup $\left(P_{t}\right)$ is strongly continuous in $C_{b}^{\text {strict }}\left(H_{b w}\right)$. Let $\mathscr{G}$ denote the generator of $\left(P_{t}\right)$ in $C_{b}^{\text {strict }}\left(H_{b w}\right)$. Then the space $\mathscr{F} C_{b}^{\infty}\left(A^{\star}\right)$ is a core for $\mathscr{G}$ and for every $\phi \in \mathscr{F} C_{b}^{\infty}\left(A^{\star}\right)$,

$$
\mathscr{G} \phi(x)=\frac{1}{2} \operatorname{Tr}\left(B^{\star} D^{2} \phi(x) B\right)+\left\langle x, A^{\star} D \phi(x)\right\rangle_{H}+\left(b_{V}, D \phi(x)\right) .
$$

\section{Proof of Theorem 2.4}

We recall first a basic fact about the conditional Gaussian measures.

3.1. Conditional Gaussian measures. Let $\left(H_{1},\langle\cdot, \cdot\rangle_{H_{1}}\right)$ and $\left(H_{2},\langle\cdot, \cdot\rangle_{H_{1}}\right)$ be two real separable Hilbert spaces and let $(X, Y)$ be a Gaussian vector defined on a probability space $(\Omega, \mathscr{F}, \mathbb{P})$ and taking values in $H_{1} \times H_{2}$. Let

$$
m_{X}=\mathbb{E} X \quad \text { and } \quad m_{Y}=\mathbb{E} Y .
$$

The covariance operator $C_{X}$ of $X$ is determined by the equation

$$
\mathbb{E}\left\langle X-m_{X}, h\right\rangle_{H_{1}}\left\langle X-m_{X}, k\right\rangle_{H_{1}}=\left\langle C_{X} h, k\right\rangle_{H_{1}}, \quad h, k \in H_{1},
$$

and a similar condition determines the covariance $C_{Y}$ of $Y$. The covariance operator $C_{X Y}: H_{1} \rightarrow H_{2}$ is defined by the condition

$$
\left\langle C_{X Y} h, k\right\rangle_{H_{2}}=\mathbb{E}\left\langle X-m_{X}, h\right\rangle_{H_{1}}\left\langle Y-m_{Y}, k\right\rangle_{H_{2}}, \quad h \in H_{1}, k \in H_{2},
$$

and then $C_{X Y}^{*}=C_{Y X}$. For a linear closable operator $G$ on $H_{i}, i=1,2$, the closure of $G$ will be denoted by $\bar{G}$. For a symmetric and compact operator $K: H_{i} \rightarrow H_{i}$ we will denote by $K^{-1}$ its pseudo inverse. For the convenience of the reader we present the following known result (see. e.g. [19]).

Theorem 3.1. The following hold.

(a) We have

$$
\operatorname{Im}\left(C_{Y X}\right) \subset \operatorname{Im}\left(C_{X}^{1 / 2}\right),
$$

and the operator $K:=C_{X}^{-1 / 2} C_{Y X}$ is of Hilbert-Schmidt type on $H_{2}$ and $K^{*}=$ $\overline{C_{X Y} C_{X}^{-1 / 2}}$.

(b) We have

$$
\mathbb{E}(Y \mid X)=m_{Y}+K^{*} C_{X}^{-1 / 2}\left(X-m_{X}\right), \quad \mathbb{P}-\text { a.s. }
$$

(c) The conditional distribution of $Y$ given $X$ is Gaussian $\mathscr{N}\left(\mathbb{E}(Y \mid X), C_{Y \mid X}\right)$, where

$$
C_{Y \mid X}:=C_{Y}-K^{*} K
$$

Moreover, the random variables $K^{*} C_{X}^{-1 / 2} X$ and $\left(Y-K^{*} C_{X}^{-1 / 2} X\right)$ are independent. 
3.2. Proof of Theorem 2.4. The proof of the theorem is divided into a sequence of lemmas.

Fix $y \in H$. Putting $\mathbb{P}=\mathbb{P}^{y}, X=Z(s)$, and $Y=Z(t)$ in (3.1) we find that for any $y \in H$ and any $0<s \leq t$,

$$
K(s, t, y):=Q^{-1 / 2}(s, y) Q^{\star}(s, t, y)
$$

is a well defined Hilbert-Schmidt operator on $H$, where $K(s, t, y)$ is the covariance operator defined in (2.1).

Lemma 3.2. (1) For any $s, t$ such that $0 \leq s \leq t$ the operator-valued mappings $x \mapsto Q(s, t, x)$ and $x \mapsto K(s, t, x)$ are constant in $x \in H$. Putting $Q(t)=Q(t, t, 0)$ and $K(s, t)=K(s, t, 0)$ we have

$$
Q(t-s)=Q(t)-K^{\star}(s, t) K(s, t)
$$

and

$$
K(s, t)=Q^{-1 / 2}(s) Q^{\star}(s, t) .
$$

(2) For any $s, t$ such that $0 \leq s \leq t$ the operator $L(t, s):=K^{\star}(s, t) Q^{-1 / 2}(s)$ with the domain $\operatorname{Im}\left(Q^{1 / 2}(s)\right)$ has a unique extension to a bounded linear operator $L(t, s): H \rightarrow H$ and for any $x, y \in H$,

$$
m(t-s, x)=m(t, y)-L(t, s) m(s, y)+L(t, s) x .
$$

Moreover,

$$
m(t-s, m(s, y))=m(t, y), \quad 0 \leq s \leq t, y \in H .
$$

Proof. Invoking Theorem 3.1 we find that for any $0<s<t$ and $y \in H$ the following equality holds $\mathbb{P}^{y}$-a.s.:

$$
\begin{aligned}
& \mathbb{E}^{y}\left(\left(Z(t)-\mathbb{E}^{y}(Z(t) \mid Z(s))\right) \otimes\left(Z(t)-\mathbb{E}^{y}(Z(t) \mid Z(s))\right) \mid Z(s)\right) \\
& \quad=Q(t, y)-K^{\star}(s, t, y) K(s, t, y) .
\end{aligned}
$$

Applying the Markov property to (3.7) we obtain for $0<s<t$,

$$
Q(t-s, x)=Q(t, y)-K^{\star}(s, t, y) K(s, t, y) \text { for } \mu(s, y)-\text { a.e. } x \text {. }
$$

For any $h, k \in H$,

$\langle Q(t-s, x) h, k\rangle_{H}=\langle Q(t, y) h, k\rangle_{H}-\left\langle K^{\star}(s, t, y) K(s, t, y) h, k\right\rangle_{H}$ for $\mu(s, y)-$ a.e. $x$.

Therefore the function $x \mapsto\langle Q(t-s, x) h, k\rangle_{H}$ is constant on a dense set and by Hypothesis 2.1 is continuous on $H$. It follows that equality (3.9) holds for every $x \in H$. Finally, for all $h, k \in H$ the function

$$
y \rightarrow\left\langle K^{\star}(s, t, y) K(s, t, y) h, k\right\rangle_{H}
$$

is constant in $y \in H$. Thus (3.3) holds.

Invoking Theorem 3.1 and the first part of the proof we obtain for $0<s<t$ and $y \in H$,

$$
\mathbb{E}^{y}(Z(t) \mid Z(s))=m(t, y)+K^{\star}(s, t, y) Q^{-1 / 2}(s)(Z(s)-m(s, y)), \quad \mathbb{P}^{y}-a . s .
$$

Then the Markov property yields for $0<s<t$,

(3.10) $m(t-s, x)=m(t, y)+K^{\star}(s, t, y) Q^{-1 / 2}(s)(x-m(s, y)) \quad$ for $\quad \mu(s, y)-a . e . x$.

Putting $x-m(s, y)=z \in H$ equation (3.10) takes the form

$$
m(t-s, z+m(s, y))=m(t, y)+K^{\star}(s, t, y) Q^{-1 / 2}(s) z \quad \text { for } \quad \nu_{s}-\text { a.e. } z,
$$


where $\nu_{s}=\mathscr{N}(0, Q(s))$. The measurable linear operator $K^{\star}(s, t, y) Q^{-1 / 2}(s)$ is well defined linear on a dense linear space $\operatorname{Im}\left(Q^{1 / 2}(s)\right)$. By Hypothesis 2.1 the operator

$$
K^{\star}(s, t, y) Q^{-1 / 2}(s)
$$

has a unique extension to a bounded linear operator $L(t, s, y)$ on $H$. Therefore, for any $u, v \in H$ and $x=Q^{1 / 2}(s) u$ and $z=Q^{1 / 2}(s) v$ we have

$$
m(t-s, x)-m(t-s, z)=K^{\star}(s, t, y)(u-v) .
$$

Hence $K^{\star}(s, t, y)$ is constant in $y \in H$ and (3.5) easily follows. Putting $x=m(s, y)$ in equation (3.5) we obtain (3.6). We have also proved that

$$
L(t, s)=L(t, s, 0)=\overline{K^{\star}(s, t) Q^{-1 / 2}(s)} .
$$

Lemma 3.3. For $0 \leq s \leq t$ we have $L(t, s)=L(t-s)$ and $(L(t))$ is a strongly continuous semigroup on $H$ and

$$
Q(s, t, x)=L(t-s) Q(s), \quad x \in H, 0 \leq s \leq t .
$$

Proof. For any $x, z \in H$, 3.5 yields

$$
m(t-s, x)-m(t-s, z)=L(t, s)(x-z) .
$$

Therefore,

$$
L(t, s) x=m(t-s, x)-m(t-s, 0)
$$

depends on $t-s$ only and there exists an operator-valued mapping, still denoted by $L$, such that

$$
L(t, s)=L(t-s), \quad 0 \leq s \leq t .
$$

Invoking (3.12) we obtain

$$
\begin{aligned}
L(s+t)(x-y) & =m(s+t, x)-m(s+t, y) \\
& =m(s, m(t, x))-m(s, m(t, y))=L(s)(m(t, x)-m(t, y)) \\
& =L(s) L(t)(x-y) .
\end{aligned}
$$

Finally,

$$
L(s+t) x=L(s) L(t) x, \quad x \in H
$$

and

By Hypothesis 2.1.

$$
L(t) x=m(t, x)-m(t, 0) .
$$

$$
\lim _{t \downarrow 0}\langle L(t) x, h\rangle_{H}=\langle x, h\rangle_{H}, \quad h \in H .
$$

Since $t \mapsto m(t, y)$ is weakly continuous for every $y \in H$, we obtain

$$
\sup _{t \leq T}|L(t) y|_{H} \leq C_{T}(y) .
$$

Therefore, the semigroup property of $(L(t))$ and a well known result (see e.g. [21], Theorem 1.4, p. 44) imply

$$
\lim _{t \downarrow 0} L(t) x=x, \quad x \in H,
$$

which proves that $(L(t))$ is a $C_{0}$-semigroup on $H$. The last part of the lemma follows immediately from (3.4) and (3.11). 
Lemma 3.4. There exist $\lambda_{0}, C>0$ such that

$$
|m(t, 0)|_{H} \leq C \mathrm{e}^{\lambda_{0} t}, \quad t \geq 0 .
$$

Proof. We have

$$
m(t, x)=m(t, y)-L(t) y+L(t) x,
$$

and therefore the $H$-valued mapping

$$
g(t):=m(t, y)-L(t) y
$$

is independent of $y \in H$. Putting $m(t, 0)=g(t)$ it is easy to check that

$$
g(t+s)=L(t) g(s)+g(t), \quad s, t \geq 0 .
$$

For any $k \geq 1$ equation (3.13) yields

$$
g(k)=\sum_{i=0}^{k-1} L(i) g(1) .
$$

Since $(L(t))$ is a $C_{0}$-semigroup in $H$ there exist finite $M$ and $\lambda_{0}>0$ such that

$$
\|L(t)\|_{L(H)} \leq M \mathrm{e}^{\lambda_{0} t}, \quad t \geq 0,
$$

where $\|\cdot\|_{L(H)}$ denotes the operator norm on the space $L(H)$ of bounded linear operators on $H$. Therefore,

$$
|g(k)|_{H} \leq\left(\sum_{i=0}^{k-1}\|L(i)\|_{L(H)}\right)|g(1)|_{H} \leq\left(\sum_{i=0}^{k-1} M \mathrm{e}^{\lambda_{0} i}\right)|g(1)|_{H} .
$$

Let

$$
c:=\frac{M}{\mathrm{e}^{\lambda_{0}}-1}|g(1)|_{H} .
$$

Now, take any $t=k+s$ where $k \geq 0$ and $s \in[0,1)$. Then

$$
g(t)=g(k+s)=L(k) g(s)+g(k),
$$

and hence

$$
|g(t)|_{H} \leq M \mathrm{e}^{\lambda_{0} k} \sup _{s \leq 1}|g(s)|_{H}+c \mathrm{e}^{\lambda_{0} k} \leq C \mathrm{e}^{\lambda_{0} t},
$$

and the lemma follows.

Lemma 3.5. There exists $b_{V} \in V$ such that

$$
m(t, 0)=\int_{0}^{t} L_{V}(s) b_{V} \mathrm{~d} s .
$$

Proof. Let $g(t)=m(t, 0)$. For $\lambda>\lambda_{0}$ the function

$$
\widehat{g}(\lambda)=\int_{0}^{+\infty} \mathrm{e}^{-\lambda t} g(t) \mathrm{d} t
$$

is well defined by Lemma 3.4. Taking the Laplace transform of both sides of equation (3.13) we obtain for $\lambda>\lambda_{0}$,

$$
\mathrm{e}^{\lambda s} \int_{0}^{+\infty} \mathrm{e}^{-\lambda(t+s)} g(t+s) \mathrm{d} t-\widehat{g}(\lambda)=(\lambda-A)^{-1} g(s),
$$

hence

$$
\mathrm{e}^{\lambda s}\left(\widehat{g}(\lambda)-\int_{0}^{s} \mathrm{e}^{-\lambda u} g(u) \mathrm{d} u\right)-\widehat{g}(\lambda)=(\lambda-A)^{-1} g(s) .
$$


Therefore,

$$
\frac{\mathrm{e}^{\lambda s}-1}{s} \widehat{g}(\lambda)-\frac{\mathrm{e}^{\lambda s}}{s} \int_{0}^{s} \mathrm{e}^{-\lambda u} g(u) \mathrm{d} u=(\lambda-A)^{-1}\left(\frac{g(s)}{s}\right) .
$$

The left-hand side of the above equality converges to

$$
b_{H}:=\lambda \widehat{g}(\lambda)
$$

for $s \downarrow 0$, and thereby the $H$-valued mapping $\frac{g(s)}{s}$ has a limit $b_{V}:=\left(\lambda-A_{V}\right) b_{H}$ in $V$ for $s \downarrow 0$. More precisely,

$$
b_{V}=\lim _{s \downarrow 0} \frac{g(s)}{s}=\left(\lambda-A_{V}\right)(\lambda \widehat{g}(\lambda)) .
$$

Invoking again equation (3.13) we obtain

$$
\lim _{s \downarrow 0} \frac{g(s+t)-g(t)}{s}=\lim _{s \downarrow 0}\left(L_{V}(t) \frac{g(s)}{s}\right)=L_{V}(t) b_{V},
$$

where the convergence holds in $V$. Finally,

$$
\frac{\mathrm{d} g}{\mathrm{~d} t}(t)=L_{V}(t) b_{V}
$$

and the lemma easily follows.

Let us recall that

$$
L(t-s)=K^{\star}(s, t) Q^{-1 / 2}(s) .
$$

Thus (3.3) can be written in the form

$$
Q(t+s)=Q(t)+L(t) Q(s) L^{\star}(t), \quad s, t \geq 0 .
$$

Note that if $R: H \rightarrow H$ is a trace class operator such that $R=R^{\star} \geq 0$, then it gives rise to an operator $\tilde{R}: V^{\prime} \rightarrow V$ which is again of trace class, and $\tilde{\tilde{R}}=\tilde{R}^{\star} \geq 0$. In the sequel we will use the same notation $R$ for both operators. For any bounded $R: H \rightarrow H$ let

$$
\mathscr{T}(t) R=L(t) R L^{\star}(t) .
$$

It may be checked that $(\mathscr{T}(t))$ is a $C_{0}$-semigroup on the space of trace-class operators on $H$. Let $\left(L_{V}(t)\right)$ denote an extension of $(L(t))$ to $V$. Then (see Lemma 2.3) $L_{V}^{\star}(t)$ can be identified with the restriction of $L^{\star}(t)$ to $V^{\prime}$. Let $\mathscr{E}$ denote the separable Banach space of symmetric trace-class operators $R: V^{\prime} \rightarrow V$ endowed with the nuclear norm. The dual space $\mathscr{E}^{\prime}$ can be identified as the space of bounded operators from $V$ to $V^{\prime}$ and the duality form $(\cdot, \cdot)$ on $\mathscr{E}^{\prime} \times \mathscr{E}$ is given by

$$
(P, R)=\operatorname{Tr}(R P)
$$

see pp. 34 and 65 of [1] for details. It is easy to see that the adjoint semigroup acting on $\mathscr{E}^{\prime}$ has the form

$$
\mathscr{T}^{\star}(t) R=L_{V}^{\star}(t) R L_{V}(t), \quad R: V \rightarrow V^{\prime} .
$$


Lemma 3.6. There exists a $B \in L(H, V)$ such that $B B^{\star} \in \mathscr{E}$, for any $t>$ $0, B^{\star} L_{V}^{\star}(t)$ has an extension to a Hilbert-Schmidt operator on $H$, the mapping $(0,+\infty) \ni s \rightarrow B^{\star} L_{V}^{\star}(s) \in L_{(H S)}(H, H)$ is continuous, and (1.4), (2.3), (2.4) hold.

Proof. Using the same arguments as in the proof of Lemma 3.4 we can show that there exist $\lambda_{0}, C>0$ such that

$$
\|Q(t)\|_{\mathscr{E}} \leq C \mathrm{e}^{\lambda_{0} t}, \quad t \geq 0 .
$$

Therefore, for any $\lambda>\lambda_{0}$ the operator

$$
\widehat{Q}(\lambda)=\int_{0}^{+\infty} \mathrm{e}^{-\lambda t} Q(t) \mathrm{d} t
$$

where the integral is the Bochner integral in $E$. Let $\mathscr{A}$ denote the generator of the semigroup $(\mathscr{T}(t))$ on $\mathscr{E}$. In view of (3.14) we can apply the same arguments as in the proof of Lemma 3.5 to obtain

$$
\lim _{s \downarrow 0}(\lambda-\mathscr{A})^{-1} \frac{1}{s} Q(s)=\lambda \widehat{Q}(\lambda),
$$

where the convergence holds in $\mathscr{E}$. Let us recall that for $h, k \in V^{\prime}$ a bounded operator $h \otimes k: V \rightarrow V^{\prime}$ is defined by the formula

$$
(h \otimes k) x=\langle h, x\rangle k, \quad x \in V .
$$

For $u, v \in \operatorname{Dom}(A) \subset V$ we have $u \otimes v: V^{\prime} \rightarrow V$. Moreover, $u \otimes u \in \operatorname{Dom}(\mathscr{A})$ and

$$
\mathscr{A}(u \otimes u)=u \otimes(A u)+(A u) \otimes u .
$$

Similarly, if $u \in V^{\prime}$, then $u \otimes u: V \rightarrow V^{\prime}, u \otimes u \in \operatorname{Dom}\left(\mathscr{A}^{\star}\right)$ and

$$
\mathscr{A}^{\star}(u \otimes u)=u \otimes\left(A^{\star} u\right)+\left(A^{\star} u\right) \otimes u .
$$

Recall that $(\cdot, \cdot)$ is the duality form on $\mathscr{E} \times \mathscr{E}^{\prime}$. By (3.16) , for $h \in H \subset V$ and $u \in V^{\prime}$,

$$
\begin{aligned}
\left(h \otimes h, \mathscr{A}^{\star}(u \otimes u)\right) & =\left(h \otimes h, u \otimes\left(A^{\star} u\right)+\left(A^{\star} u\right) \otimes u\right) \\
& =\left(h \otimes h, u \otimes\left(A^{\star} u\right)\right)+\left(h \otimes h,\left(A^{\star} u\right) \otimes u\right) \\
& =\langle h, u\rangle_{H}\left\langle h, A^{\star} u\right\rangle_{H}+\left\langle h, A^{\star} u\right\rangle_{H}\langle h, u\rangle_{H} \\
& =2\langle h, u\rangle_{H}\left\langle h, A^{\star} u\right\rangle_{H} \\
& =2\left\langle(h \otimes h) u, A^{\star} u\right\rangle_{H},
\end{aligned}
$$

where the first step follows from (3.16). Therefore, by the properties of the nuclear norm on $E$ we find that for any $R \in \mathscr{E}$ and $u \in V^{\prime}$,

$$
\left(R, \mathscr{A}^{\star}(u \otimes u)\right)=2\left\langle R u, A^{\star} u\right\rangle_{H}
$$

By (3.15) we have

$$
\lim _{s \downarrow 0}\left((\lambda-\mathscr{A})^{-1} \frac{1}{s} Q(s), P\right)=(\lambda \widehat{Q}(\lambda), P)
$$


for any $P \in \mathscr{E}^{\prime}$. Taking $P=\left(\lambda-\mathscr{A}^{\star}\right)(u \otimes u)$ with $u \in \operatorname{Dom}\left(A_{V}^{\prime}\right) \subset V^{\prime}$ and using (3.17) we obtain

$$
\begin{aligned}
\lim _{s \downarrow 0}\left\langle\frac{1}{s} Q(s) u, u\right\rangle_{H} & =\left(\lambda \widehat{Q}(\lambda),\left(\lambda-\mathscr{A}^{\star}\right)(u \otimes u)\right) \\
& =\lambda\langle\lambda \widehat{Q}(\lambda) u, u\rangle_{H}-2 \lambda\left\langle\widehat{Q}(\lambda) u, A^{\star} u\right\rangle_{H} .
\end{aligned}
$$

The bilinear form

$$
\begin{aligned}
\mathscr{B}(u, v) & :=\lim _{s \downarrow 0}\left\langle\frac{1}{s} Q(s) u, v\right\rangle_{H} \\
& =\lambda\langle\lambda \widehat{Q}(\lambda) u, v\rangle_{H}-\lambda\left\langle\widehat{Q}(\lambda) u, A^{\star} v\right\rangle_{H}-\lambda\left\langle\widehat{Q}(\lambda) v, A^{\star} u\right\rangle_{H}
\end{aligned}
$$

is well defined for $u, v \in V^{\prime}=\operatorname{Dom}\left(A^{\star}\right)$ and symmetric as a limit in $s \downarrow 0$ of symmetric bilinear forms. Moreover,

$$
|\mathscr{B}(u, v)| \leq c|u|_{V^{\prime}}|v|_{V^{\prime}} \quad \text { and } \quad \mathscr{B}(u, u) \geq 0 .
$$

Therefore, there is a symmetric operator $S: V^{\prime} \rightarrow V$ such that

$$
\mathscr{B}(u, v)=(S u, v), \quad u, v \in V^{\prime} .
$$

Since (see Lemma 2.3) $A$ has a bounded extension to an operator from $H$ to $V$ and $A^{\star}$ is a bounded linear operator from $V^{\prime}$ to $H$,

$$
S=\lambda^{2} \widehat{Q}(\lambda)-\lambda A \widehat{Q}(\lambda)-\lambda \widehat{Q}(\lambda) A^{\star} .
$$

Hence $S$ is a nuclear operator from $V^{\prime}$ to $V$ as $\widehat{Q}(\lambda)$ is a nuclear operator on $H$. Then (see Lemma 2.3) there exists a bounded linear operator $B$ from $H$ to $V$ such that $S=B B^{\star}$.

Invoking (3.14), we obtain that for $u \in V^{\prime}$,

$$
\begin{aligned}
\frac{\mathrm{d}}{\mathrm{d} t}\langle Q(t) u, u\rangle_{H} & =\mathscr{B}\left(L^{\star}(t) u, L^{\star}(t) u\right) \\
& =\left(S L^{\star}(t) u, L^{\star}(t) u\right)=\left\langle B^{\star} L_{V}^{\star}(t) u, B^{\star} L_{V}^{\star}(t) u\right\rangle_{H} .
\end{aligned}
$$

Hence, using polarisation, we obtain (1.5).

Let $t>0$. Since $Q(t)$ is a covariance operator, it is nuclear on $H$, and as a consequence of the identity above, $\left(B^{\star} L_{V}^{\star}(s)\right)^{\star} B^{\star} L_{V}^{\star}(s)$ has for almost all $s$, a unique extension to a nuclear operator on $H$. Since

$\infty>\operatorname{Trace} Q(t)=\int_{0}^{t} \operatorname{Trace}\left(B^{\star} L_{V}^{\star}(s)\right)^{\star} B^{\star} L_{V}^{\star}(s) \mathrm{d} s=\int_{0}^{t}\left\|B^{\star} L_{V}^{\star}(s)\right\|_{L_{(H S)}(H, H)}^{2} \mathrm{~d} s$.

Thus for almost all $s, B^{\star} L_{V}^{\star}(s)$ has an extension to a Hilbert-Schmidt operator on $H$. Taking into account the semigroup property of $\left(L_{V}^{\star}(t)\right)$ we obtain that $B^{\star} L_{V}^{\star}(s)$ has an extension to a Hilbert-Schmidt operator on $H$ for all $s$ and the mapping $(0,+\infty) \ni s \rightarrow B^{\star} L_{V}^{\star}(s) \in L_{(H S)}(H, H)$ is continuous. Combining this with (1.5) we obtain (2.3) and (2.4). 


\section{Proof of Theorem 2.7}

Let $\left(\Omega, \mathscr{F},\left(\mathscr{F}_{t}\right), Z,\left\{\mathbb{P}^{x}: x \in H\right\}\right)$ be a Markov family with the transition kernels $\mu(t, x), x \in H, t \geq 0$. Let $x \in H$. We define a $V$-valued and $\left(\mathscr{F}_{t}\right)$-adapted process

$$
M(t):=Z(t)-Z(0)-t b_{V}-\int_{0}^{t} A_{V} Z(s) \mathrm{d} s .
$$

It is easy to check that for each $x, \mathbb{P}^{x}$-a.s.,

$$
M(t)=Z(t)-m(t, x)-\int_{0}^{t} A_{V}(Z(s)-m(s, x)) \mathrm{d} s .
$$

Then $\mathbb{P}^{x}$-a.s.

$$
\begin{aligned}
\mathbb{E}^{x}( & \left.M\left(t_{2}\right) \mid \mathscr{F}_{t_{1}}\right) \\
= & \mathbb{E}^{x}\left(Z\left(t_{2}\right)-m\left(t_{2}, x\right) \mid Z\left(t_{1}\right)\right) \\
& -\int_{0}^{t_{1}} A_{V}(Z(s)-m(s, x)) \mathrm{d} s-\int_{t_{1}}^{t_{2}} A_{V} \mathbb{E}^{x}\left(Z(s)-m(s, x) \mid Z\left(t_{1}\right)\right) \\
= & L\left(t_{2}-t_{1}\right)\left(Z\left(t_{1}\right)-m\left(t_{1}, x\right)\right) \\
& -\int_{0}^{t_{1}} A_{V}(Z(s)-m(s, x)) \mathrm{d} s-\int_{t_{1}}^{t_{2}} A_{V} L\left(s-t_{1}\right)\left(Z\left(t_{1}\right)-m\left(t_{1}, x\right)\right) \mathrm{d} s \\
= & Z\left(t_{1}\right)-m\left(t_{1}, x\right)-\int_{0}^{t_{1}} A_{V}(Z(s)-m(s, x)) \mathrm{d} s \\
= & M\left(t_{1}\right) .
\end{aligned}
$$

Let $M_{h}(t)=\langle M(t), h\rangle_{H}$ for $h \in V^{\prime}=\operatorname{dom}\left(A^{\star}\right)$. Then on each filtered probability space $\left(\Omega, \mathscr{F},\left(\mathscr{F}_{t}\right), \mathbb{P}^{x}\right), M_{h}$ is a continuous Gaussian martingale with $M_{h}(0)=0$. Since the martingale is Gaussian, we have

$$
\left\langle M_{h}\right\rangle_{t}=\mathbb{E}^{x}\left|M_{h}(t)\right|^{2} .
$$

It remains to compute $\mathbb{E}^{x}\left|M_{h}(t)\right|^{2}$. To this end we write

$$
\begin{aligned}
\left|M_{h}(t)\right|^{2}= & \langle Z(t)-m(t, x), h\rangle_{H}^{2}+\left(\int_{0}^{t}\left\langle Z(s)-m(s, x), A^{\star} h\right\rangle_{H} \mathrm{~d} s\right)^{2} \\
& -2 \int_{0}^{t}\langle Z(t)-m(t, x), h\rangle_{H}\left\langle Z(s)-m(s, x), A^{\star} h\right\rangle_{H} \mathrm{~d} s .
\end{aligned}
$$

Then

$$
\begin{aligned}
\mathbb{E}^{x}\left|M_{h}(t)\right|^{2}= & \langle Q(t) h, h\rangle_{H} \\
& +\int_{0}^{t} \int_{0}^{t} \mathbb{E}^{x}\left\langle Z(s)-m(s, x), A^{\star} h\right\rangle_{H}\left\langle Z(u)-m(u, x), A^{\star} h\right\rangle_{H} \mathrm{~d} u \mathrm{~d} s \\
& -2 \int_{0}^{t}\left\langle L(t-s) Q(s) A^{\star} h, h\right\rangle_{H} \mathrm{~d} s .
\end{aligned}
$$

Since $\langle Q(t) h, h\rangle_{H}$ can be written in the form

$$
\langle Q(t) h, h\rangle_{H}=\int_{0}^{t}\left\langle B^{\star} L_{V}^{\star}(t-s) h, B^{\star} L_{V}^{\star}(t-s) h\right\rangle_{H} \mathrm{~d} s
$$


we obtain

$$
\begin{aligned}
\frac{d}{d t} \mathbb{E}^{x}\left|M_{h}(t)\right|^{2}= & 2\left\langle Q(t) h, A^{\star} h\right\rangle_{H}+\left|B^{\star} h\right|_{H}^{2} \\
& +\int_{0}^{t} \mathbb{E}^{x}\left\langle Z(s)-m(s, x), A^{\star} h\right\rangle_{H}\left\langle Z(t)-m(t, x), A^{\star} h\right\rangle_{H} \mathrm{~d} s \\
& +\int_{0}^{t} \mathbb{E}^{x}\left\langle Z(t)-m(t, x), A^{\star} h\right\rangle_{H}\left\langle Z(u)-m(u, x), A^{\star} h\right\rangle_{H} \mathrm{~d} u \\
& -2 \int_{0}^{t}\left\langle L(t-s) Q(s) A^{\star} h, A^{\star} h\right\rangle_{H} \mathrm{~d} s-2\left\langle Q(t) h, A^{\star} h\right\rangle_{H} \\
= & \left|B^{\star} h\right|_{H}^{2} .
\end{aligned}
$$

Let $\left\{e_{i}: i \geq 1\right\}$ denote an orthonormal basis spanning the closure $H_{0}$ of $\operatorname{Im}\left(B^{\star}\right)$ in $H$ and let $B^{\star} h_{i}=e_{i}$. Then under each $\mathbb{P}^{x}$, the processes

$$
\beta_{i}^{x}(t)=M_{h_{i}}(t), \quad i \geq 1,
$$

are standard independent Brownian motions; hence we can define a generalised process $W$ on $H_{0}$ such that $M_{h}(t)=W(t)\left[B^{\star} h\right]$. Moreover, $W$ is a cylindrical Wiener process on $H_{0}$ defined on each filtered probability space $\left(\Omega, \mathscr{F},\left(\mathscr{F}_{t}\right), \mathbb{P}^{x}\right)$ and such that for any $h \in \operatorname{dom}\left(A^{\star}\right)$,

$$
\langle Z(t), h\rangle_{H}=\langle Z(0), h\rangle_{H}+\int_{0}^{t}\left\langle Z(s), A^{\star} h\right\rangle_{H} \mathrm{~d} s+\int_{0}^{t}\left(b_{V}, h\right) \mathrm{d} s+W(t)\left[B^{\star} h\right] .
$$

Now, Theorem 2.7 follows from the equivalence of weak and mild solutions established in [5]; see also 8].

Remark 4.1. Let us recall an alternative, but typical for stochastic differential equations, description of the Markov family with transition kernels $\{\mu(t, x): x \in H$, $t \geq 0\}$. A starting point is a filtered probability space $(\Omega, \mathscr{F},(\mathscr{F} t), \mathbb{P})$ and a family $\left\{Z^{x}: x \in H\right\}$ of $H$-valued and $\left(\mathscr{F}_{t}\right)$-adapted stochastic processes such that for every $x \in H$, we have $\mathbb{P}\left(Z^{x}(0)=x\right)=1$, and for all $t, s \geq 0$ and Borel set $\Gamma \subset H$,

$$
\mathbb{P}\left(Z^{x}(t+s) \in \Gamma \mid \mathscr{F}_{s}\right)=\mu\left(t, Z^{x}(s)\right)(\Gamma), \quad \mathbb{P}-\text { a.s. }
$$

Then with a minor modification of the proof we can show that for each initial value $x \in H$ there exists a cylindrical Wiener process $W^{x}$ on $H_{0}$ such that

$$
Z^{x}(t)=L(t) x+\int_{0}^{t} L_{V}(t-s) b_{V} \mathrm{~d} s+\int_{0}^{t} L_{V}(t-s) B \mathrm{~d} W^{x}(s), \quad t \geq 0, \mathbb{P}-\text { a.s. }
$$

To see that in this case the process $W$ may depend on $x$ it is enough to consider the one-dimensional Wiener processes

$$
Z^{x}(t)=x+\beta(t) \cos x+\tilde{\beta}(t) \sin x, \quad x \in \mathbb{R}, t \geq 0,
$$

where $\beta$ and $\tilde{\beta}$ are independent real-valued Brownian motions.

\section{Proof of Theorem 2.8}

It was proved in 20] that $P_{t} C_{b}\left(H_{b w}\right) \subset C_{b}\left(H_{b w}\right)$. Let $B_{r} \subset H$ denote the centered closed ball of radius $r$. Let us recall that bounded sets of $H_{b w}$ are precisely the bounded sets of the norm topology and the sets $B_{r}$ are compact in $H_{b w}$. The proof is a simple modification of the proof of Theorem 4.2 in [15]. Let us note here that by definition of the $b w$-topology (see [13]) the space $H_{b w}$ is a $k$-space 
and therefore the space $C_{b}^{\text {strict }}\left(H_{b w}\right)$ is complete. We provide details only for the proof of strong continuity. To this end it is enough to show that for every weakly sequentially continuous $\phi: H \rightarrow \mathbb{R}$ and every $r>0$,

$$
\lim _{t \rightarrow 0} \sup _{x \in B_{r}}\left|P_{t} \phi(x)-\phi(x)\right|=0 .
$$

Assume that this convergence does not hold. Then there exist $\varepsilon>0$, a sequence $t_{n} \rightarrow 0$ and a sequence $\left(x_{n}\right) \subset B_{r}$ such that

$$
\left|P_{t_{n}} \phi\left(x_{n}\right)-\phi\left(x_{n}\right)\right|>\varepsilon \text {. }
$$

Therefore

$$
\int_{H}\left|\phi\left(m\left(t_{n}, x_{n}\right)+y\right)-\phi\left(x_{n}\right)\right| \mu\left(t_{n}\right)(\mathrm{d} y)>\varepsilon
$$

where by Theorem 2.4 the measure $\mu(t)(\mathrm{d} y):=\mathscr{N}\left(0, Q\left(t, x_{n}\right)\right)(\mathrm{d} y)$ is independent of $n$. Since $\left(x_{n}\right)$ is bounded, by the Banach-Alaoglu theorem it is weakly compact. Taking a subsequence we can assume that $\left(x_{n}\right)$ converges weakly to a certain $x \in H$. Since $\phi$ is bounded and $\phi\left(x_{n}\right) \rightarrow \phi(x)$, for $n$ large enough we have

$$
\int_{H}\left|\phi\left(m\left(t_{n}, x_{n}\right)+y\right)-\phi(x)\right| \mu\left(t_{n}\right)(\mathrm{d} y) \geq \frac{\varepsilon}{2} .
$$

Hypothesis 2.1 yields

$$
\left\langle m\left(t_{n}, 0\right), h\right\rangle_{H} \rightarrow 0, \quad \forall h \in H,
$$

and as $\left|L^{\star}\left(t_{n}\right) h-h\right|_{H} \rightarrow 0$, we have

$$
\lim _{n \rightarrow \infty}\left\langle x_{n}, L^{\star}\left(t_{n}\right) h\right\rangle_{H}=\langle x, h\rangle_{H}, \quad \forall h \in H .
$$

Thus

$$
\left\langle m\left(t_{n}, x_{n}\right), h\right\rangle_{H}=\left\langle x_{n}, L^{\star}\left(t_{n}\right) h\right\rangle_{H}+\left\langle m\left(t_{n}, 0\right), h\right\rangle_{H} \longrightarrow\langle x, h\rangle_{H}, \quad \forall h \in H .
$$

Let $\left(Y_{n}\right)$ be the sequence of random variables such that each $Y_{n}$ has the distribution $\mathscr{N}\left(m\left(t_{n}, x_{n}\right), Q\left(t_{n}\right)\right)$. Then $\left(Y_{n}\right)$ converges in probability to the constant vector $x$. On the other hand, for $n$ large enough (5.2) yields

$$
\mathbb{E}\left|\phi\left(Y_{n}\right)-\phi(x)\right| \geq \frac{\varepsilon}{2},
$$

which leads to a contradiction.

We pass now to the proof of the second part of the theorem. It is easy to check that for any $\phi \in \mathscr{F} C_{b}^{\infty}\left(A^{\star}\right)$ formula (2.7) holds and by the definition of the space $\mathscr{F} C_{b}^{\infty}\left(A^{\star}\right)$ the function $\mathscr{L} \phi$ is a well defined element of $C_{b}^{\text {strict }}\left(H_{b w}\right)$. The proof that $\mathscr{F} C_{b}^{\infty}\left(A^{\star}\right)$ is a core for $\mathscr{L}$ is an easy modification of the proofs of Lemma 4.4 and Theorem 4.5 in [15] and is thus omitted.

\section{EXample: Heat equation with boundary noise}

We will consider here a stochastic PDE with boundary noise introduced in 7 ] (see also [9]) and studied in [1, 2], and [14]:

$$
\begin{cases}\frac{\partial Z}{\partial t}(t, \xi)=\frac{\partial^{2} Z}{\partial \xi^{2}}(t, \xi), & t>0, \xi>0 \\ Z(t, 0)=\dot{\beta}(t), & t>0, \\ Z(t, \xi)=x(\xi), & \xi>0,\end{cases}
$$


where $\beta=(\beta(t))$ is a real-valued Brownian motion. Using some heuristic arguments Da Prato and Zabczyk [7] defined a solution to (6.1) as the process

$$
Z(t):=\mathrm{e}^{t \Delta} x+(1-\Delta) \int_{0}^{t} \mathrm{e}^{(t-s) \Delta} D \mathrm{~d} \beta(s),
$$

where $\Delta=\mathrm{d}^{2} / \mathrm{d} \xi^{2}$ is the Laplace operator in $L^{2}([0,+\infty) ; \mathrm{d} \xi)$ with Dirichlet boundary conditions, and $D: \mathbb{R} \rightarrow L^{2}(0,+\infty ; \mathrm{d} \xi)$, given by the formula

$$
\mathbb{R} \ni r \rightarrow \operatorname{Dr}(\xi)=r \mathrm{e}^{-\xi} \in L^{2}([0,+\infty) ; \mathrm{d} \xi),
$$

is the so-called Dirichlet map. Alòs and Bonaccorsi [1] defined a solution to (6.1) in the following way:

$$
Z(t, \xi)=\int_{0}^{+\infty} G(t, \xi, \eta) x(\eta) \mathrm{d} \eta+\int_{0}^{t} \frac{\partial G}{\partial \eta}(t-s, \xi, 0) \mathrm{d} \beta(s),
$$

where

$$
G(t, \xi, \eta)=\frac{1}{\sqrt{4 \pi t}}\left(\mathrm{e}^{-|\xi-\eta|^{2} / 4 t}-\mathrm{e}^{-|\xi+\eta|^{2} / 4 t}\right), \quad \xi, \eta \geq 0 .
$$

Note that $G$ is the kernel of the semigroup $\left(\mathrm{e}^{t \Delta}\right)$. In 2 a systematic study of general stochastic boundary value problems using the concept of weak solution was initiated, and in particular the equivalence between Da Prato and Zabczyk, and Alòs and Bonaccors definitions is established. In 2] the solution lives in the space of generalised functions, whereas in 14, it is shown that (6.1) defines a Markov family in the weighted space $H=L^{2}([0,+\infty) ; \rho(\xi) \mathrm{d} \xi)$, where $\rho(\xi)=\min \left(1, \xi^{1+\alpha}\right)$ with $\alpha \in(0,1)$. Below, a result from [14] is presented in the framework introduced in our paper. Recall that the space $V$ and the extension $L_{V}$ were introduced in Definition 2.2 and Lemma 2.3 , respectively.

Proposition 6.1. (i) $\left\{Z^{x}: x \in H\right\}$ defined by (6.2) is a Gauss-Markov family in $H$.

(ii) The heat semigroup $\left(\mathrm{e}^{t \Delta}\right)$ has an extension to an analytic $C_{0}$-semigroup $(L(t))$ on $H$.

(iii) The law $\mathscr{L}\left(Z^{x}(t)\right)$ of $Z^{x}(t)$ is Gaussian $\mathscr{N}(m(t, x), Q(t))$, where $m$ and $Q$ are given by (1.4) and (1.5), respectively, with $b_{V}=0$, the operator $B$ given by the formula

$$
B x=\left(1-A_{V}\right) f \frac{\langle x, f\rangle_{H}}{|f|_{H}}, \quad f(\xi)=\mathrm{e}^{-\xi},
$$

and $A_{V}$ being the generator of $\left(L_{V}(t)\right)$.

(iv) There exists a cylindrical Wiener process $W$ on $H_{0}=\operatorname{linspan}\{f\}$ such that

$$
Z^{x}(t)=L(t) x+\int_{0}^{t} L_{V}(t-s) B \mathrm{~d} W(s), \quad t \geq 0, x \in H .
$$

Remark 6.2. Part (ii) of the proposition has been proved in [18].

\section{Appendix A. Proof of Lemma 2.3}

Proof of (i). Let $t \geq 0$. Since $H$ is dense in $V$, to show that $L(t)$ has a bounded extension to the operator $L_{V}(t)$ on $V$ it is enough to show that there exists a constant $C_{t}$ such that

$$
|L(t) x|_{V} \leq C_{t}|x|_{V}, \quad \forall x \in H
$$


To see this, note that as $L(t) x \in H$,

$$
\begin{aligned}
|L(t) x|_{V} & =|L(t) x|_{-1}=\left|(\lambda-A)^{-1} L(t) x\right|_{H}=\left|L(t)(\lambda-A)^{-1} x\right|_{H} \\
& \leq\|L(t)\|_{L(H)}\left|(\lambda-A)^{-1} x\right|_{-1}=\|L(t)\|_{L(H)}|x|_{V},
\end{aligned}
$$

where $\|\cdot\|_{L(H)}$ is the operator norm on the space $L(H)$ of bounded linear operators on $H$. Obviously, $\left(L_{V}(t)\right)$ is a semigroup on $V$. The strong continuity follows from the fact that $\left\|L_{V}(t)\right\|_{L(V)} \leq\|L(t)\|_{L(H)}$ and that $L_{V}(t) x \rightarrow x$ as $t \rightarrow 0$ in $H$ and hence in $V$.

We are going to show first that $A$ has an extension to a bounded linear operator $\tilde{A}$ from $H$ to $V$. Then we will show that the generator $A_{V}$ of $L_{V}$ is equal to $\tilde{A}$. To see the first claim take $x \in \operatorname{dom} A$. Then

$$
|A x|_{V}=\left|(\lambda-A)^{-1} A x\right|_{H}=\left|A(\lambda-A)^{-1} x\right|_{H} \leq\left\|A(\lambda-A)^{-1}\right\|_{L(H)}|x|_{H} .
$$

Since $\operatorname{dom} A$ is dense in $H$, this proves that $A$ has a unique extension to a bounded linear operator from $H$ to $V$. Now let $A_{V}$ denote the generator of $L_{V}$. Obviously $A_{V}$ is an extension of $A$. Note that $H \subset \operatorname{dom} A_{V}$. Indeed, let $x \in H$. Then there is a sequence $\left(x_{n}\right)$ of elements of $\operatorname{dom} A$ such that $x_{n} \rightarrow x$ in $H$, and hence in $V$. Since $\tilde{A}$ is a bounded operator from $H$ to $V$, we have

$$
A_{V} x_{n}=A x_{n}=\tilde{A} x_{n} \rightarrow \tilde{A} x,
$$

where the convergence is in $V$. Since $A_{V}$ is closed, $x \in \operatorname{dom} A_{V}$ and $\tilde{A} x=A_{V} x$. Thus $A_{V}$ is an extension of $\tilde{A}$. To see that $\operatorname{dom} A_{V} \subset H$ note that $\operatorname{dom} A$ is a core for $A_{V}$. This (see e.g. [10, Theorem 1.9, p. 8) follows from the fact that $\operatorname{dom} A$ is dense in $V$ and invariant for $(L(t))$ and hence also for $\left(L_{V}(t)\right)$. Now let $x \in \operatorname{dom} A_{V}$. Then there is a sequence $\left(x_{n}\right) \subset \operatorname{dom} A$ such that $x_{n} \rightarrow x$ in $V$ and $A_{V} x_{n} \rightarrow A_{V} x$ also in $V$. Thus

$$
\lim _{n, m \rightarrow \infty}\left|\left(\lambda-A_{V}\right)\left(x_{n}-x_{m}\right)\right|_{V}=0 .
$$

But

$$
\left|\left(\lambda-A_{V}\right)\left(x_{n}-x_{m}\right)\right|_{V}=\left|x_{n}-x_{m}\right|_{H} .
$$

Hence $\left(x_{n}\right)$ converges in $H$, and consequently $x \in H$.

Proof of (ii). We are showing now the second part of the lemma. To do this note that if $x \in \operatorname{dom} A^{\star}$, then for any $y \in H$,

$$
\left|\langle x, y\rangle_{H}\right|=\left|\left\langle\left(\lambda-A^{\star}\right) x,(\lambda-A)^{-1} y\right\rangle_{H}\right| \leq\left|\left(\lambda-A^{\star}\right) x\right|_{H}|y|_{V} \cdot
$$

Thus $x$ can be extended to a linear functional on $V$, and hence $x \in V^{\prime}$. Assume now that $x \in V^{\prime}$. Then there is a constant $C$ such that for any $y \in \operatorname{dom} A$,

$$
\left|\langle x,(\lambda-A) y\rangle_{H}\right| \leq C|(\lambda-A) y|_{V}=C|y|_{H} .
$$

Hence $x \in \operatorname{dom} A^{\star}$, and the desired conclusion follows.

Proof of (iii). If $S: V^{\prime} \rightarrow V$ is non-negative and symmetric, then there exists a separable Hilbert space $H_{S}$ and an imbedding $i: H_{S} \rightarrow V$ such that $S=i \circ i^{\star}$; see for example Proposition 1.6 and the following corollary in Chapter III of [23]. Let $I: H_{S} \rightarrow H$ be the canonical isometry of $H_{S}$ on a closed subspace of $H$. Then $B=i \circ I^{\star}$ has the desired property. 


\section{ACKNOWLEDGEMENT}

The authors wish to express their gratitude to an anonymous referee for a thorough reading of the manuscript, for making valuable remarks, and for pointing out some errors.

\section{REFERENCES}

[1] E. Alòs and S. Bonaccorsi, Stochastic partial differential equations with Dirichlet white-noise boundary conditions (English, with English and French summaries), Ann. Inst. H. Poincaré Probab. Statist. 38 (2002), no. 2, 125-154, DOI 10.1016/S0246-0203(01)01097-4. MR.1899108 (2003a:60093)

[2] Z. Brzeźniak, B. Goldys, S. Peszat, and F. Russo, Second order PDEs with Dirichlet white noise boundary conditions, J. Evol. Equ. 15 (2015), no. 1, 1-26, DOI 10.1007/s00028-0140246-2. MR 3315663

[3] Z. Brzeźniak, M. Ondrejat, and J. Seidler, Invariant measures for stochastic nonlinear beam and wave equations (2012), preprint.

[4] Ph. Courrège, Sur la forme integro-différentielle des opérateurs de $C_{K}^{\infty}$ dans $C_{0}$ satisfaisant au principe du maximum, Sém. Théorie du Potentiel, Exposé 2, 1965/1966.

[5] A. Chojnowska-Michalik, Stochastic differential equations in Hilbert spaces, Probability theory (Papers, VIIth Semester, Stefan Banach Internat. Math. Center, Warsaw, 1976), Banach Center Publ., vol. 5, PWN, Warsaw, 1979, pp. 53-74. MR561468 (81j:60068)

[6] S. Cerrai, Weakly continuous semigroups in the space of functions with polynomial growth, Dynam. Systems Appl. 4 (1995), no. 3, 351-371. MR.1348505 (96h:35249)

[7] G. Da Prato and J. Zabczyk, Evolution equations with white-noise boundary conditions, Stochastics Stochastics Rep. 42 (1993), no. 3-4, 167-182. MR1291187 (95m:60088)

[8] G. Da Prato and J. Zabczyk, Stochastic equations in infinite dimensions, Encyclopedia of Mathematics and its Applications, vol. 44, Cambridge University Press, Cambridge, 1992. MR.1207136 (95g:60073)

[9] G. Da Prato and J. Zabczyk, Ergodicity for infinite-dimensional systems, London Mathematical Society Lecture Note Series, vol. 229, Cambridge University Press, Cambridge, 1996. MR.1417491 (97k:60165)

[10] E. B. Davies, One-parameter semigroups, London Mathematical Society Monographs, vol. 15, Academic Press, Inc. [Harcourt Brace Jovanovich, Publishers], London-New York, 1980. MR.591851(82i:47060)

[11] A. Defant and K. Floret, Tensor norms and operator ideals, North-Holland Mathematics Studies, vol. 176, North-Holland Publishing Co., Amsterdam, 1993. MR.1209438 (94e:46130)

[12] D. Duffie, D. Filipović, and W. Schachermayer, Affine processes and applications in finance, Ann. Appl. Probab. 13 (2003), no. 3, 984-1053, DOI 10.1214/aoap/1060202833. MR1994043 (2004g:60107)

[13] N. Dunford and J. T. Schwartz, Linear operators. Part I, General theory; with the assistance of William G. Bade and Robert G. Bartle; reprint of the 1958 original; Wiley Classics Library, John Wiley \& Sons, Inc., New York, 1988. MR.1009162 (90g:47001a)

[14] G. Fabbri and B. Goldys, An LQ problem for the heat equation on the halfline with Dirichlet boundary control and noise, SIAM J. Control Optim. 48 (2009), no. 3, 1473-1488, DOI 10.1137/070711529. MR2496985 (2010h:93133)

[15] B. Goldys and M. Kocan, Diffusion semigroups in spaces of continuous functions with mixed topology, J. Differential Equations 173 (2001), no. 1, 17-39, DOI 10.1006/jdeq.2000.3918. MR.1836243 (2002c:60097)

[16] K. Itô, Infinite-dimensional Ornstein-Uhlenbeck processes, Stochastic analysis (Katata/Kyoto, 1982), North-Holland Math. Library, vol. 32, North-Holland, Amsterdam, 1984, pp. 197-224, DOI 10.1016/S0924-6509(08)70394-5. MR780759 (86i:60117)

[17] O. Kallenberg, Foundations of modern probability, 2nd ed., Probability and its Applications (New York), Springer-Verlag, New York, 2002. MR1876169(2002m:60002)

[18] N. V. Krylov, The heat equation in $L_{q}\left((0, T), L_{p}\right)$-spaces with weights, SIAM J. Math. Anal. 32 (2001), no. 5, 1117-1141, DOI 10.1137/S0036141000372039. MR1828321 (2002a:35092) 
[19] A. Mandelbaum, Linear estimators and measurable linear transformations on a Hilbert space, Z. Wahrsch. Verw. Gebiete 65 (1984), no. 3, 385-397, DOI 10.1007/BF00533743. MR731228 (85h:62030)

[20] B. Maslowski and J. Seidler, Strong Feller solutions to SPDE's are strong Feller in the weak topology, Studia Math. 148 (2001), no. 2, 111-129, DOI 10.4064/sm148-2-2. MR.1881256 (2003f:60112)

[21] A. Pazy, Semigroups of linear operators and applications to partial differential equations, Applied Mathematical Sciences, vol. 44, Springer-Verlag, New York, 1983. MR710486 (85g:47061)

[22] B. Schmuland and W. Sun, On the equation $\mu_{t+s}=\mu_{s} * T_{s} \mu_{t}$, Statist. Probab. Lett. 52 (2001), no. 2, 183-188, DOI 10.1016/S0167-7152(00)00235-2. MR1841407 (2002i:60007)

[23] N. N. Vakhania, V. I. Tarieladze, and S. A. Chobanyan, Probability distributions on Banach spaces, Translated from the Russian and with a preface by Wojbor A. Woyczynski. Mathematics and its Applications (Soviet Series), vol. 14, D. Reidel Publishing Co., Dordrecht, 1987. MR:1435288(97k:60007)

School of Mathematics and Statistics, The University of Sydney, Sydney 2006, AusTRALIA

E-mail address: Beniamin.Goldys@sydney.edu.au

Institute of Mathematics, Polish Academy of Sciences, Św. Tomasza 30/7, 31-027 Cracow, Poland

E-mail address: napeszat@cyf-kr.edu.pl

Current address: Institute of Mathematics, Jagiellonian University, Łojasiewicza 6, 30-348 Kraków, Poland

Institute of Mathematics, Polish Academy of Sciences, Śniadeckich 8, 00-950 Warsaw, POLAND

E-mail address: J.Zabczyk@impan.pl 